\title{
Evaluation des pertes d'agneaux et de chevreaux par abattage des femelles gravides
}

\author{
Y. Manjeli ${ }^{1}$ R.M. Njwe ${ }^{1}$ J. Tchoumboué ${ }^{1}$ S. Abba ${ }^{2}$ \\ A. Teguia ${ }^{1}$
}

\begin{abstract}
Mots-clés
Brebis - Chèvre - Agneau - Chevreau Perte - Abattage - Gestation Cameroun.
\end{abstract}

\begin{abstract}
Résumé
Les pertes d'agneaux et de chevreaux dues à l'abattage des femelles gravides ont été évaluées entre 1993 et 1995 aux abattoirs de Garoua et de Maroua, Cameroun. Sur un total de 3150 chèvres et 1757 brebis examinées après abattage, 1216 chèvres soit 38,6 p. 100 et 1040 brebis soit 59,2 p. 100 étaient en état de gravidité plus ou moins avancé. Les pertes étaient respectivement de l'ordre de 0,6 chevreau et 1 agneau par femelle abattue. Ces pertes économiques sont importantes et coïncident avec des déficits en viande qui ne cessent de s'aggraver. Des mesures urgentes doivent être envisagées pour en diminuer l'ampleur. Cependant, des mesures efficaces de prévention risquent d'être complexes si le contexte socio-économique encore axé sur l'économie de subsistance et le système d'élevage extensif des petits ruminants reste prédominant.
\end{abstract}

\section{INTRODUCTION}

Le Cameroun compte environ 3,5 millions de petits ruminants dont 45 p. 100 d'ovins et 55 p. 100 de caprins. Les rôles de cet élevage villageois sont multiples : élevage tirelire permettant de subvenir aux besoins urgents de l'éleveur (argent, cadeaux, sacrifices...).

Malgré des effectifs relativement élevés ayant une importance socio-économique, la productivité du cheptel des petits ruminants est faible et cela pour des raisons diverses : mortalité des jeunes avant et post-sevrage évaluée à 40-50 p. 100, déficiences alimentaires quantitatives et qualitatives saisonnières, problèmes pathologiques où le parasitisme gastro-intestinal joue un rôle prépondérant. Cependant, dans l'évaluation des causes de cette faible productivité, les pertes des jeunes dues à l'abattage d'un grand nombre de femelles gravides sont rarement prises en compte. L'évaluation de ces pertes, liées aux abattages des mères gestantes, a fait l'objet de quelques études au Cameroun (1, 2, 3, $4,5,7,8,9)$. Les études sont, pour la plupart, limitées dans la zone du Sud Cameroun, aucune ne faisant référence aux caprins.

L'objectif du présent travail est d'évaluer l'importance des pertes par abattage des brebis et chèvres gestantes dans la région septentrionale du Cameroun.

\section{MATERIEL ET METHODES}

L'étude s'est déroulée de février 1993 à septembre 1995 aux abattoirs de Garoua et Maroua. Un total de 3150 chèvres et 1757 bre-

\footnotetext{
1. Département de zootechnie, Faculté d'agronomie et des sciences agricoles, Université de Dschang, BP 222 Dschang, Cameroun

2. Ministère de l'agriculture, Yaoundé, Cameroun
}

bis ont été examinées après abattage. En cas de gestation, l'âge du fœtus était établi par un examen systématique :

- des ovaires (présence du corps jaune) ;

- de l'état du développement de l'utérus et des poches placentaires ;

- du fœtus avec examen du pelage, ongles, dentition, poids, longueur du corps pour en fixer l'âge (6).

\section{RESULTATS}

Comme le montre le tableau I, le taux de femelles gravides dans les deux espèces est relativement élevé, juste un peu moins de la moitié et presque les deux tiers des femelles abattues respectivement pour les chèvres et les brebis. L'analyse de la répartition de l'âge de la gestation des femelles gravides montre que les abattages de la première moitié de la gestation (0-2 mois) représentent 60,2 p. 100 et 69,3 p. 100 des chèvres et des brebis gravides abattues. Au cours de la deuxième moitié (2-5 mois), bien que les pourcentages soient relativement faibles, ils restent néanmoins élevés pour une période où les signes de la gravidité sont morphologiquement perceptibles et permettent ainsi un diagnostic précis.

Les pertes en jeunes résultant de l'abattage des femelles gestantes sont d'autant plus importantes que la proportion des gestations gémellaires est élevée. Le tableau II montre la répartition des femelles gravides en fonction du type de gestation et met en évidence le fait que la proportion des femelles abattues en état de gestation gémellaire représente environ les deux tiers du total des femelles gestantes abattues dans les deux espèces. Si la répartition des types de gestation observés reflète la réalité, les pertes des jeunes sont alors respectivement de 1992 et 1732 chevreaux et agneaux soit 0,6 chevreau et 1 agneau par chèvre et par brebis abattue. 


\section{Tableau I}

Répartition des chèvres et brebis gravides abattues en fonction de la durée de la gestation

\section{Durée de la gestation} (mois)

Femelles gravides abattues

\begin{tabular}{|lcccc|}
\hline & \multicolumn{2}{c}{ Chèvres } & \multicolumn{2}{c|}{ Brebis } \\
\hline & Nombre & $\% *$ & Nombre & $\% *$ \\
$0-1$ & 421 & 34,6 & 430 & 41,3 \\
$1-2$ & 324 & 26,6 & 293 & 28,2 \\
$2-3$ & 235 & 19,3 & 237 & 22,8 \\
$3-4$ & 199 & 16,3 & 49 & 4,7 \\
$4-5$ & 37 & 3,1 & 31 & 3,0 \\
\hline Total femelles & & & & \\
gravides abattues & 1216 & $38,6 *$ & 1040 & $59,2 * *$ \\
\hline
\end{tabular}

* Pourcentage par rapport aux femelles gravides abattues

** Pourcentage par rapport aux femelles abattues contrôlées

\section{Tableau II}

Répartition des chèvres et brebis gravides abattues par types de gestation

\begin{tabular}{lcccc|} 
& \multicolumn{3}{c}{ Femelles gravides abattues } \\
\cline { 2 - 5 } Types de gestation & \multicolumn{2}{c}{ Chèvres } & \multicolumn{2}{c|}{ Brebis } \\
& Nombre & $\%$ & Nombre & $\%$ \\
& 440 & 36,2 & 348 & 33,5 \\
Simples & 776 & 63,8 & 692 & 66,5 \\
Doubles & 1216 & 100,0 & 1040 & 100,0 \\
\hline Total & & & &
\end{tabular}

\section{DISCUSSION}

Les résultats de cette étude montrent l'importance des pertes des petits ruminants par suite de l'abattage des femelles gestantes. Celles-ci doivent d'ailleurs être évaluées à la hausse puisque les femelles abattues avant l'implantation et la placentation ou à un stade de gravidité très précoce n'ont pas été prises en compte.

Dans la zone considérée, l'élevage des petits ruminants de type essentiellement extensif implique que dans son troupeau l'éleveur n'a pas la maitrise du processus de la reproduction qui a lieu au hasard des rencontres mâles - femelles. L'éleveur ignore ainsi l'état de gravidité de ses femelles jusqu'à un stade avancé de celle-ci.
Si la vente des animaux à un stade précoce de la gestation peut être compréhensible, on ne s'explique pas le taux d'abattage encore relativement élevé des femelles se situant dans la deuxième moitié (3-5 mois) de gestation, leur aspect physique dénotant clairement leur condition aussi bien auprès de l'éleveur qui les vend que du boucher qui les abat.

L'abattage des femelles gestantes pourrait donc s'expliquer non pas par l'ignorance de l'état physiologique des animaux, mais davantage par le contexte socio-économique de l'élevage des petits ruminants qui est considéré comme un élevage de subsistance tirelire destiné à la vente pour résoudre les besoins urgents quel que soit l'état physiologique des animaux. De ce fait, on peut présager de la complexité et de la difficulté qu'il y aurait à élaborer et à mettre en pratique des mesures de prévention de l'abattage des femelles gravides. Pourtant il faut les envisager. En effet, les pertes des petits par abattage ajoutées à celles des petits nés évaluées actuellement à 40-50 p. 100 et à d'autres types de pertes (mortalité des adultes...), montrent un manque à gagner non négligeable pour l'économie nationale qu'il serait préférable d'estimer pour pouvoir ensuite envisager les possibilités, au moins, de le limiter.

\section{CON CLUSION}

Il ressort de cette étude que la proportion des femelles gravides vendues et abattues quel que soit le stade de la gravidité est relativement élevée et atteint 40 et 50 p. 100 du total des femelles abattues respectivement pour les espèces caprines et ovines. Ces abattages entraînent des pertes énormes en petits évaluées respectivement à 0,6 chevreau et 1 agneau par femelle abattue .

A l'échelon national, il s'agit de pertes économiques importantes contre lesquelles des mesures urgentes doivent être envisagées pour en diminuer l'ampleur.

Cependant, tant que le système d'élevage extensif restera prédominant et que les petits ruminants seront élevés dans le contexte d'économie de subsistance, les mesures efficaces de prévention de ces pratiques désastreuses risquent d'être complexes et difficiles à mettre en œuvre. Pourtant il faut les envisager en urgence étant données les pertes qu'elles entrainent pour l'économie nationale.

\section{BIBLIO GRAPHIE}

1. MUKASA-MUGERWA E., TEKELYE B., 1988. The reproductive performance of Ethiopian Highland sheep. Anim. Reprod. Sci., 17: 95-102.

2. NDI C., TAMBI N.E., AGARITH N.W., 1993. Reducing calf wastage from the slaughtering of pregnant cows in Cameroon. Revue mond. Zootec., $77: 4-5$.

3. N SEKAYARENZE J., 1988. Contribution à la détermination de l'âge du fœtus de brebis Peul-Peul du Sénégal (A propos de 138 fœtus récoltés aux abattoirs de Dakar). Thèse doct. médecine vétérinaire $n^{\circ} 39$, Ecole inter-états de Sciences médicales et vétérinaire, Université Dakar, Sénégal.

4. TCHOUMBOUE J., 1984. Pertes de veaux par abattage de vaches gestantes. Cas particulier de l'abattoir de Yaoundé (Cameroun). Revue Elev. Méd. vét. Pays trop., 37 (1) : 70-72.

5. TCHOUMBOUE J., 1988. Note sur l'abattage de brebis gestantes et les pertes d'agneaux au Cameroun. Revue Elev. Méd. vét. Pays trop., 41 (4) : 461-462.

6. TCHOUMBOUE J., 1989. Pertes de porcelets par abattage des truies gestantes au Cameroun. Revue Elev. Méd. vét. Pays trop., 42 (4) : 589-590. 
7. WILSO N R.J., TRAORE A., 1988. Livestock production in central $M$ ali. Reproductive performance and reproductive wastage in ruminants in the agro-pastoral system. Theriogenology, 29 (4): 931-944.

8. WOSU L.O., 1988. Calf wastage through slaughtering of pregnant cows in Enugu abattoir, Nigeria. Revue Elev. Méd. vét. Pays trop., 41 (1) : $97-98$.
9. WOSU L.O., DIBUA E.C., 1992. Lamb and kid wastage through slaughtering of pregnant ewes and goats at Enugu and N sukka abattoirs in Anambra State, Nigeria. In: Rey B., Lebbie S.H.B., Reynolds L. eds. Small ruminant research and development in Africa. Proceedings of the first biennial conference of the African small Ruminant Research Network, 10-14 December 1990, Nairobi, Kenya. Nairobi, Kenya, ILRAD.

Reçu le 6.5.96, accepté le 28.10 .96

\section{Summary}

Manjeli Y., Njwe R.M., Tchoumboué J., Abba S., Teguia A. Loss evaluation of lambs and kids through slaughtering of pregnant females

Loss of lambs and kids through slaughtering of pregnant ewes and goats at Garoua and Maroua abattoirs, Cameroon, was evaluated between 1993 and 1995. O ut of 3150 goats and 1757 ewes slaughtered, 1216 goats $(38.6 \%)$ and 1040 ewes $(59.2 \%)$ were pregnant. Losses were estimated at 0.6 kid and 1 lamb per slaughtered female respectively. These losses have consequential economic impacts at a time when meat shortage is growing. Urgent measures should be taken to reduce the magnitude of the problem. However, efficient preventive measures might be complicated if the socioeconomic context, still based on subsistence economy and small ruminant extensive husbandry, remains prevalent.

Key words: Ewe - Goat - Lamb - Kid - Loss - Slaughtering Pregnancy - Cameroon.

\section{Resumen}

Manjeli Y., Njwe R.M., Tchoumboué J., Abba S., Teguia A. Evaluación de las pérdidas de corderos y cabritos por el sacrificio de hembras gestantes

Se evaluaron las pérdidas de corderos y cabritos debidas al sacrificio de hembras gestantes, entre 1993 y 1995 en los mataderos de Garoua y Maroua, Camerún. Sobre un total de 3150 cabras y de 1757 ovejas examinadas post-mortem, 1 216 cabras, es decir 38,6 p. 100 y 1040 ovejas, sea 59,2 p. 100 se encontraban en estado de gravidez más o menos avanzada. Las pérdidas son, respectivamente, del orden de 0,6 cabrito y de un cordero por hembra sacrificada. Estas pérdidas económicas son importantes y coinciden con los déficits en carne, los cuales no cesan de agravarse. Deben tomarse medidas urgentes para disminuir la importancia. Sin embargo, las medidas eficaces de prevención podrían resultar complejas, si el contexto socio-económico, aún centrado sobre la economía de subsistencia y el sistema de crianza extensivo de los pequeños rumiantes, sigue predominando.

Palabras clave : O veja - Cabra - Cordero - Cabrito - Pérdida Sacrificio - Gestación - Camerún. 